\title{
Participación social en sistemas de salud fragmentados: ¿una relación virtuosa?*
}

\section{Social Participation in Fragmented Health Systems: a Virtuous Relationship?}

\section{Participação social em sistemas de saúde fragmentados: um relacionamento virtuoso?}

Fecha de recepción: 04-11-15 Fecha de aceptación: 24-02-16 Disponible en línea: 03-05-16 doi:10.11144/Javeriana.rgyps15-30.psss

Cómo citar este artículo:

Jaime SF. Participación social en sistemas de salud fragmentados: ¿Una relación virtuosa? Rev. Gerenc. Polít. Salud. 2016; 15(30): 38-56. http://dx.doi.org/10.11144/Javeriana.rgyps15-30.psss

Sergio Fernando Jaime** 


\section{Resumen}

Los sistemas de salud se enfrentan a diferentes obstáculos que amenazan su correcto desempeño. Uno de ellos es su fragmentación. Como contrapartida, la participación social en salud es mencionada con la misma intensidad como fenómeno integrador. Este estudio tuvo como objetivo proporcionar un marco más concreto sobre la compleja relación entre ambos emergentes de los sistemas de salud y para ello utilizó diferentes métodos: una revisión de la literatura, un enfoque cualitativo para definir las dimensiones de análisis y, en un tercer momento, técnicas de ensayos clásicos (ensayo expositivo) para argumentar cada dimensión. Como producto final se definieron doce tesis que en conjunto se proponen explicar satisfactoriamente este fenómeno e inferir líneas de trabajo para mejorar de manera efectiva el funcionamiento de los sistemas de salud.

Palabras clave: sistemas de salud/tendencias, fragmentación, participación social, participación ciudadana, complejidad, sistemas integrados

\section{Abstract}

Health systems face different obstacles that threaten their proper performance. One such obstacle is the fragmentation of health systems. In return, the social participation in health is mentioned with the same intensity that integrating phenomenon. This study aimed to provide a more concrete framework on the complex relationship between these factors emerging health systems and for that use different methods: A review of literature, a qualitative approach to define the dimensions of analysis and in a third time a classic technique assay (expository essay) to argue each dimension. The final product 12 theses were defined, and together aim to satisfactorily explain this phenomenon and propose lines of work that effectively improve the performance of health systems.

Keywords: health systems/trends, fragmentation, social participation, complexity, citizen participation, system integration

\section{Resumo}

Os sistemas de saúde enfrentam-se a diferentes obstáculos que ameaçam seu desempenho adequado. Um deles é sua fragmentação. Como contrapartida, a participação social em saúde é mencionada com igual intensidade como fenómeno integrador. Este estudo objetivou fornecer quadro mais concreto sobre o complexo relacionamento entre ambos os emergentes dos sistemas de saúde e para isso utilizou diferentes métodos: uma revisão da literatura, um enfoque qualitativo para definir as dimensões de análise e, em uma terceira vez, técnicas de ensaios clássicos (ensaio expositivo) para argumentar cada dimensão. Como produto final definiram-se doze teses que em conjunto propõem-se explicar satisfatoriamente este fenómeno e inferir linhas de trabalho para melhorar de maneira efetiva o funcionamento dos sistemas de saúde.

Palavras-chave: sistemas de saúde/tendências, fragmentação, participação social, participação cidadã, complexidade, sistemas integrados 


\section{Introducción}

Los sistemas de salud constituyen uno de los pilares esenciales de las sociedades modernas. A pesar de ello, frecuentemente no pueden abordar de manera satisfactoria el objeto que justifica su existencia (las necesidades de salud de una sociedad); ya sea porque su rectoría y gobernanza se ve limitada, su prestación de servicios es insuficiente, no satisfacen las expectativas de la gente, o bien la complejidad de la problemática resiste acciones lineales destinadas a someterlas (1-5).

Para explicar estas dificultades de los sistemas de salud en conseguir sus objetivos esenciales, usualmente se hace referencia a tendencias que atentan contra su correcto funcionamiento, y dentro de estas tendencias la fragmentación (6) es una de las más mencionadas, un término comúnmente definido como "lo opuesto a integración" (7). Pero cuando se intenta ser más concreto, para un abordaje de estudio más correcto, se observa una enorme variabilidad conceptual y operativa en la utilización del concepto. La definición más utilizada, "coexistencia de muchas unidades o entidades no integradas en la red de servicios de salud" (8), se suma a múltiples definiciones que la utilizan en un mismo sentido: describir negativamente a los servicios y sistemas de salud (9-17).

\section{La participación social en salud tampoco} tiene una única definición, pero usualmente se entiende como "la intervención de los grupos organizados de la comunidad en las deliberaciones, en la toma de decisiones y en el control de los procesos de promoción y atención de la salud, así como la asunción de responsabilidades y compromisos derivados de la facultad decisoria" (18). $\mathrm{Su}$ pronunciamiento se asocia a un valor socialmente positivo, pero las posibilidades de interpretación también abren un amplio abanico de posibilidades entre lo enunciado y lo que efectivamente se implementa, siendo esto último influenciado por contextos, subjetividades y utilitarismos varios (19-23).

A pesar de esta ambigüedad conceptual, se propone sistemáticamente a la participación social en salud como una herramienta integradora de los sistemas de salud y la ubican como un pilar fundamental de los sistemas basados en la estrategia de Atención Primaria de la Salud (24-26). Pero tanto la participación social en salud como la fragmentación (y su opuesto la integración) son fenómenos complejos, por lo que su relación también será de naturaleza compleja. Por ello, el análisis no sería satisfactorio si únicamente se incluyesen justificaciones que asumieran una relación causa-efecto positivo y debería incluir otras posibilidades, incluso las que proponen cierto escepticismo en sus fundamentos. El presente trabajo tiene como objetivo establecer de qué manera se plantea o puede plantearse la relación entre participación social y fragmentación en los sistemas de salud, y las posibles conclusiones que surjan de este análisis que sean relevantes para el estudio de los sistemas de salud.

\section{Material y métodos}

Este trabajo utilizó una metodología mixta para su desarrollo: revisión, análisis cualitativo de la información relevada de los textos y técnicas de ensayo para definir líneas argumentativas, desde una perspectiva fundamentalmente cualitativa. $\mathrm{Al}$ momento de realizar el documento inicial, base del desarrollo ulterior del trabajo, el autor estaba finalizando el curso "Desarrollo de capacidades para la renovación de la Atención Primaria de la Salud", organizado por la Organización Panamericana de la Salud, que profundizaba sobre algunos aspectos que se consideran en este trabajo y en el cual se propiciaba una amplia discusión, lo cual fue 
una influencia para la selección de los temas principales (participación social en salud y fragmentación de los sistemas de salud). En la primera etapa se realizó una revisión clásica: una búsqueda bibliográfica del término participación social y fragmentación en la base de datos LILACs (seleccionando solo textos accesibles completos y artículos en español e inglés) y luego una búsqueda más detallada en revistas de salud pública indexadas en el sitio Scielo Public Health a octubre del 2013. Del conjunto de textos seleccionados se elaboró un marco teórico, lo cual en principio también puede considerarse una etapa de preanálisis en la metodología cualitativa para el análisis de contenidos.

En un segundo momento, sobre este conjunto de textos se realizó una exploración del material para identificar dimensiones de análisis. Los pasos que se siguieron para esta etapa fueron:

- Se definió una consigna inicial: identificar factores o elementos que relacionen a la fragmentación con la participación social en salud.

- Se realizó una la lectura superficial de los textos seleccionados y del marco teórico.

- Se descompuso la consigna inicial en tantas unidades de contexto y unidades de registro como se iban detectando en el texto.

- $\quad$ Se las enumero.

- Se definieron categorías e identificadores para cada categoría.

- Luego de una segunda lectura, se reorganizo y se redujeron los datos.

De esa manera, quedaron constituidas doce dimensiones de análisis: 1) el enfoque como fenómenos sociológicos; 2) la variabi- lidad de conceptos; 3) medio o fin; 4) grupos humanos que se integran exitosamente; 5) las relaciones humanas en salud; 6) conflictividad; 7) las trayectorias posibles; 8) el contexto; 9) liderazgo; 10) teoría y práctica; 11) sistemas complejos; 12) análisis histórico. Todas ellas con una breve descripción de cada categoría del porqué se relacionan con la fragmentación y la participación social en los sistemas de salud.

Estas doce dimensiones y su marco teórico fueron la matriz que sirvió como disparador para un tercer momento, donde cada una de ellas fue el eje para desarrollar líneas argumentativas que, siguiendo la técnica de ensayos expositivos, permitió definir doce tesis. Para este último momento, se recurrió a bibliografía complementaria, textos clásicos y literatura gris que permitiesen reforzar cada una de las líneas argumentativas, complementándose con las licencias propias de los ensayos: definir una relación entre premisas y una conclusión que definiría la tesis principal a través de la argumentación.

\section{Desarrollo}

\section{Doce tesis sobre la relación entre fragmentación y participación social}

Tesis 1: los sistemas de salud están destinados a ser herramientas de integración social. La participación social en salud tiene como objetivo devolver al sistema de salud a su estado natural

Parsons (27) hace referencia a que el desarrollo de una sociedad es equivalente al nivel de autarquía que desarrolle y considera a la sociedad como un sistema de intercambio o relacional entre cuatro subsistemas (el sistema político, el social, el económico y el 
cultural), cada uno de ellos especializado en una función. Los sistemas sociales, dentro de los cuales se encuentran los sistemas de salud, tienen como función especializada la unidad de acciones de los individuos en el campo de la salud, y a través de ello aportan al desarrollo de una sociedad mejor. La pregunta subsiguiente a estas afirmaciones sería por qué es necesario un subsistema cuya función principal sea la unidad de acción, y la respuesta está dada en la constitución actual de la sociedad moderna y posmoderna, que "erosiona, debilita y separa en forma progresiva al individuo con la sociedad" (28), debido a la división social del trabajo y a la creciente autonomía de los individuos. Esto hace necesario una solidaridad social que actúe como fuerza integradora de los individuos a la sociedad. La cohesión es parte de esa solidaridad necesaria, una evolución de las solidaridades mecánicas premodernas (relacionadas más que todo con lazos territoriales, tradicionales y grupales), y se refiere tanto a los mecanismos de inclusión social instituidos como a los comportamientos y valoraciones de los sujetos que conforman la sociedad. La participación social, en la medida que aporte a una mayor cohesión social (que puede entenderse también como una disminución de la fragmentación, aunque el término cohesión tiene otros alcances) será un elemento que aportará al mejor desempeño del sistema de salud y por ende a la construcción de una mejor sociedad (29-31).

Tesis 2: la participación social y la fragmentación no son términos homogéneos. Su abordaje dependerá de qué definición previa se elija

La participación social y la fragmentación son términos polisémicos. En participación social en salud básicamente se proponen dos variantes conceptuales. La primera orienta su pensamiento al interrogante de cómo hacer que las personas tomen control de sus propias vidas; la segunda ve a la participación como recurso, tratando de que esta sea un instrumento de mejor distribución de los recursos (32,33). Ambas corrientes ideológicas se transforman en la práctica en diferentes modalidades de participación, que van desde ver al individuo como un objeto que con su presencia legitima, hasta dispositivos más refinados de cogestión, decisión compartida y control ciudadano (34-36).

La fragmentación también incluye múltiples dimensiones en su concepto, todas ellas con una connotación negativa, por lo cual la medición de su impacto en salud será diferente de acuerdo a cuál de ellas se aborde: a) aquellas que enfocan su punto de vista en la coordinación entre estructuras y recursos destinados a un mismo fin (con una subdivisión dentro de esta categoría en integración vertical y horizontal, virtual y real, entre otras tipologías usadas); b) aquellas que estudian un enfoque más relacionado con un concepto integral del proceso salud-enfermedad; y c) aquellas que estudian la fragmentación del sistema de salud de otros sistemas sociales, como el político, el económico y el cultural (37-44). En el extremo opuesto a la fragmentación como fenómeno negativo existen modelos de organización sanitaria que enuncian la autonomía e independencia (o descentralización) como virtudes propias de organizaciones eficientes, y esta aparente contradicción obliga a reflexionar sobre qué lugar ocupa la fragmentación en la línea que separa a los servicios autogestionados, pero coordinados entre sî con aquellos independientes, altamente especializados y eficaces, aun cuando segmentados en su conjunto (45-46).

Esta variabilidad de términos que parecen reflejar una misma cosa, le ha restado funcionalidad al estudio y al uso de la participación social como herramienta y fin en el 
mejoramiento de las condiciones de vida de una población dada y a la fragmentación como objeto de abordaje de acciones de mejora del desempeño del sistema de salud (47). Por ello, para que la participación cumpla su objetivo como determinante de sistemas de salud menos fragmentados - y a través de un sistema de salud más integrado, un cambio social positivo-, no se debe caer en la trampa de la ambigüedad entre principios, medios y fines. Es necesario ser claros y críticamente reflexivos en los conceptos y argumentos que se esgrimen, para evitar caer en la contradicción entre objetivos enunciados y practicados, estigma de las organizaciones y sistemas modernos que resisten cambios aun cuando esto es sentido como necesario (48-49).

Tesis 3: la participación social es un ejercicio de ciudadanía. Su implementación es un objetivo en sí mismo, con independencia de su eficacia en integrar el sistema de salud

La ciudadanía se engloba dentro de la teoría de los derechos fundamentales. En sus dimensiones descriptivas y prescriptivas invoca una relación de implicancia y condicionamiento mutuo con un estado de derecho y derechos fundamentales de individuos, miembros de una sociedad libre que a través de un pacto social ejercen esta autonomía en un contexto de derechos y deberes social y jurídicamente definidos, que asumen el desafío de integrar individuos y grupos de individuos en un ámbito de tolerancia y pluralismo (50).

La participación en salud y el ejercicio de ciudadanía quizás tengan en el reclamo de derechos legalmente constituidos — tanto en el ámbito de la litigación judicial como en los cuerpos legislativos - una de las facetas más evidentes. No está claro que a través de la litigación se llegue a un mejor desempeño o una mayor integración del sistema, pero es innegable que en un Estado de derecho no es concebible la imposibilidad de reclamos judiciales ante situaciones de conflicto que atenten contra el derecho individual, a pesar de ver en muchos casos a los impactos de los fallos judiciales carentes de un análisis del beneficio o perjuicio social que reproducen $(51,52)$.

El concepto de ciudadanía no se basa exclusivamente en derechos y deberes fundamentales de los miembros de una sociedad, también se basa en propiciar igualdad de oportunidades, lo cual en el caso de aquellos con mayores desventajas sociales implicaría esfuerzos mayores por parte del Estado $(53,54)$. Paradójicamente, es en movimientos reivindicativos donde la participación demuestra una eficacia en el cumplimiento de objetivos sociales o comunitarios sentidos y en una mayor integración de esfuerzos (muchas veces muy bien coordinados), si bien deslegitiman al Estado como garante del derecho de los ciudadanos y devalúan el sistema político como representativo de la sociedad, entendiendo a esta forma de participación como una factor más de fragmentación social (55-57). La integración de un Estado de derecho con un diálogo social permanente y una abogacía por parte de este del cambio social positivo quizás permitan convertir en fortaleza esta situación frecuente en la realidad latinoamericana (58-63).

Tesis 4: la participación social en un contexto de fragmentación de los sistemas de salud encuentra en el capital social un término integrador

¿Por qué algunos colectivos de individuos llegan a cumplir en mayor medida sus aspiraciones a través de una construcción conjunta? Aparte del capital físico y del humano, las sociedades o colectivos presentan un tercer elemento que crea valor a través de las interacciones sociales: el capital social (64). Clásicamente, tres líneas argumentales son 
las que fundamentan la relevancia de esta variable social: a) las que profundizan en los recursos (tangibles e intangibles) que se originan de la red de relaciones en las cuales los individuos tengan capacidad de movilización y sentido de pertenencia; b) las que hacen hincapié en la función, o sea, el valor que tiene para los actores aquellos aspectos de la estructura social necesarios para perseguir sus intereses colectivos; c) las que ponen en el eje del debate al rol del individuo; cómo este pasa de ser una unidad a ser parte de un colectivo que busca el bien común de sus integrantes (65-67).

Lo relevante del capital social es que permite explorar en forma objetiva cómo las redes de relaciones que se constituyen en un colectivo (organizaciones, comunidades, sociedades, etc.) afrontan diversos desafíos: a) cómo reducir costos de transacción; b) cómo producir mayor cantidad de bienes públicos y; c) cómo constituir organizaciones de gestión de base efectivas en un contexto social positivo. Propone básicamente que existen características de las relaciones entre individuos que pueden volverse recursos (capital) para la acción. Es decir, participación, integración (de aspiraciones y esfuerzos) y efectividad social unidos en un mismo concepto (68-70).

Dado su carácter de variable objetivable, ya se identifican algunos atributos que acrecientan el capital social en las redes de relaciones. Incluye elementos como compromiso de los participantes con el tema en cuestión, la capacidad de colaborar y trabajar en equipo, el ambiente resultante de la confianza mutua, las imágenes y modelos mentales predominantes de sociedades vistos desde la solidaridad, el respeto a principios y valores consensuados, el clima de estabilidad, la satisfacción de necesidades afectivas, las posibilidades de desarrollo, las relaciones democráticas y la transparencia en las comunicaciones $(71,72)$. También fue sugerido como un intermediario entre equidad y nivel de salud, siendo actualmente reconocido como un determinante de la salud (73-75). Esta posibilidad de medición brinda una enorme base empírica como campo de estudio que relaciona participación, integración y efectividad de acciones colectivas (76).

Tesis 5: en los sistemas de salud las relaciones que se constituyen son asimétricas. La participación social podría disminuir los efectos negativos de esta asimetría, contribuyendo así al fortalecimiento institucional

Desde la salud pública, siempre se mencionó al modelo médico hegemónico como un lastre que impedía una armónica relación entre la sociedad y sus proveedores de servicios de salud $(77,78)$. ¿Pero de qué manera esta relación, siempre necesaria, podría abordarse sin perder la esencia del acto profesional como servicio de salud individual, un acto que constituye la unidad productiva básica de los sistemas de salud? (79).

La nueva economía institucional proporcionó un marco de análisis, explicando la delegación del poder de decisión en el agente (médico o gestor de salud) y considerando asimétrica esta relación, debido a una asimetría de conocimiento que impide que el principal (individuo-sociedad) tome la mejor decisión por él mismo. Por lo tanto, hay una brecha entre la función de utilidad de los decisores (prestadores de servicios finales) y la de los expuestos a sus decisiones (80). Por ello, se considera que las relaciones entre los individuos y los agentes se deberían dar en un entorno basado en una relación contractual, la cual pretende regular cuatro aspectos negativos de esta relación: comportamiento oportunista, racionalidad limitada, individualismo metodológico y comportamiento egoísta. Esta relación contractual debería buscar maximizar la función de utilidad de los individuos (o de 
la sociedad) con la de los decisores, al menor coste posible, y ahí es donde intervienen las instituciones proporcionando un marco o reglas de juego donde se privilegie el equilibrio a través de sanciones, ordenes jerarquizadas, sistemas de incentivos, sistemas de información y control, carreras profesionales, etc. Una teoría complementaria, la teoría de los costes de transacción, asume que el enfoque contractual es insuficiente (debido a la imposibilidad de regular a través de contratos todas las situaciones emergentes) y que la clave está en los dispositivos de coordinación y motivación como complementarias y sustentadoras de una relación más equilibra (81-84).

Esta revalorización del papel de institución de las organizaciones proveedoras de servicios de salud permite una nueva manera de abordar la participación social, en el contexto de sistemas de salud fragmentados. En la medida que la participación social forme parte de un contrato social-institucional y de la agenda política, que haya dispositivos formales e informales que permitan una mayor y mejor participación de la sociedad en las decisiones y el monitoreo de los sistemas de salud, que existan mecanismos para motivar a los agentes a disminuir la brecha que separa sus decisiones del interés de la sociedad-instituciones (empoderamiento), que la función de utilidad sea suficiente y los costes aceptables, la participación social en salud y la integración podrán ser una herramienta gerenciable de mejora de las aspiraciones sociales en salud (85-86).

Tesis 6: la participación social puede desarrollarse en un contexto conflictivo. En esta situación la política será el interlocutor que permita un marco de integración

No todos los procesos de participación social se manifestarán en un ámbito de intereses comunes consensuados. Cuando múltiples ac- tores participan en la construcción de una agenda, es usual que representen diferentes intereses o ideologías, y por eso inclusive se naturaliza el que en una sociedad coexistan la integración y el conflicto (87). Las comunidades mismas asumen como mecanismo de cohesión entre su pares, la pertenencia a una simbología común (territorial, cultural u otras), y por lo tanto, desde sus inicios su participación en fenómenos colectivos tendrá como objetivo reforzar sus rasgos de identidad, a veces de manera tan estricta que excluyen al diferente de forma tan intensa como cuando integran a un par (88-89).

En la Antigüedad ya se planteaba que todos los hombres y las comunidades miraban su propio bien y deseaban lo mejor para ellos en cada acción que acometían. En consecuencia, era entendible la defensa de sus intereses (90). Es por ello que aun la participación social altamente formal, como sucede en instituciones sociales mediante asambleas o cuerpos colegiados, al representar diferentes sectores, difícilmente pueda escapar de su naturaleza conflictiva (91-94).

La movilización social, las manifestaciones y protestas son - de cierta manera- espacios de participación social y signos de fragmentación social que, a pesar de su carácter combativo y menoscabo a las instituciones, es necesario su abordaje político desde el Estado como dispositivo de integración (95-96). Para ello la política, como campo, disciplina o acción humana, acepta la naturaleza polémica de las acciones humanas y a su vez brinda un marco de integración, inclusive cuando no sea un objetivo declarado, ya que instrumenta en diversas medidas cuotas de antagonismo e integración (97). Es - o debería ser- parte de la arena política, la construcción social en un contexto de antagonismos; de no serlo puede utilizarse métodos absolutos como forma de resolverlos (como puede ser el uso de la violencia). 
La política, por consiguiente, es un mecanismo regulador que crece donde hay diversidad $\mathrm{y}$ donde pueden hacerse distinciones entre las realidades sociales. De ahí su potencial aporte a la disminución de la fragmentación que ocurre en los procesos participativos en escenarios conflictivos (98-102).

Tesis 7: debido a la naturaleza compleja de la fragmentación, su relación con la participación social no es univoca y no siempre implica una mayor integración de los sistemas de salud

La participación social puede orientarse en distintos sentidos y los desenlaces pueden ser múltiples. Las iniciativas destinadas a promover procesos participativos no siempre logran una mayor integración del sistema, a pesar de que este sea un objetivo frecuentemente declarado en programas, planes y políticas de salud $(103,104)$. Un ejemplo claro lo da el proceso de reforma en México, que promovía un seguro popular de salud (estrategia de integración) e incluía en forma concreta dispositivos de participación social. Sobre ello un autor reflexiona: "[...] algunos retos de la gobernanza más importante se ubicaron en el ámbito de la participación social, cuyo efecto más negativo se refirió a la falta de coordinación entre ciudadanía, dependencias gubernamentales y organizaciones civiles [...]" (105).

En otro extremo, experiencias de descentralización y participación social en salud sugieren que la participación social también puede propiciar un modelo fragmentado en salud, creando espacios comunitarios de respuesta y gestión a problemas locales de salud, validando un sistema en el cual el bien común se limita a lo local, siendo "islas" o "agujeros" dentro del sistema sanitario, de acuerdo al desempeño alcanzado (106-112).

Asimismo, existen estrategias efectivas, destinadas a disminuir la fragmentación del sistema de salud, que no están relacionadas con fenómenos participativos ni con el fortalecimiento de la cohesión social, sino con modelos organizativos y de gestión, como algunas estrategias englobadas bajo el concepto de servicios de salud integrados, en el cual el foco se orienta a acciones políticas, técnicas y administrativas destinadas a proveer servicios de salud a aquellos que lo necesitan (13,113). Si bien se prescribe a la participación social como un atributo esencial de estas redes integradas, lejos se está de tener experiencias sólidas que fundamenten esta afirmación (114-116).

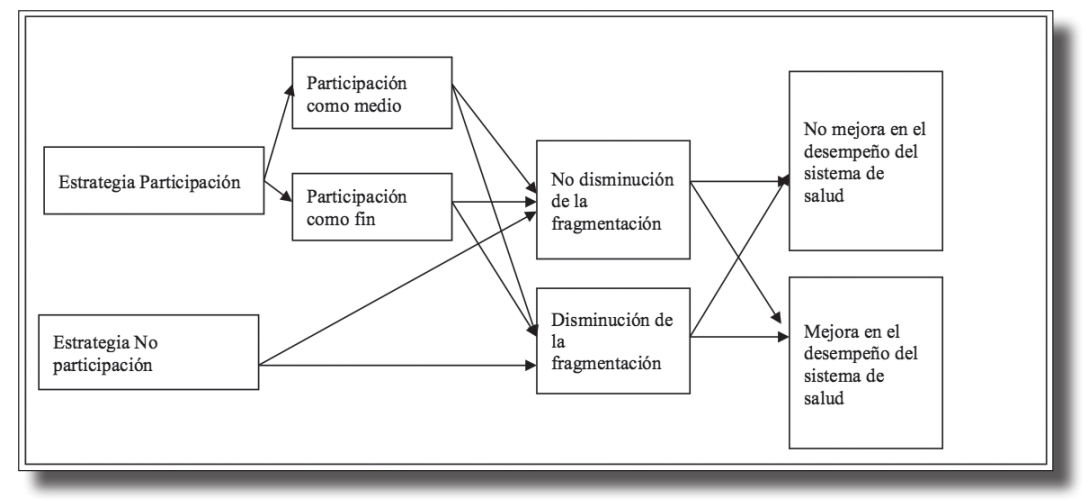

Figura 1. Las MUltiples TRAYeCtorias de La PARTICIPACION SOCIAL EN SALUd EN SISTEMAS DE SALUD FRAGMENTADOS

Fuente: elaboración propia 
Tesis 8: los valores hegemónicos determinan el modelo participativo y condicionan sus resultados

Existe un modelo de participación social en salud para cada territorio, fruto de las características y dinámicas internas del sistema (la fragmentación es una de esas características) y reactivo a un contexto social superior que lo condiciona constantemente. La participación social es un reflejo de la voluntad de los individuos en un marco de uso de sus derechos. Si bien es lógico y moralmente aceptable que se promuevan valores como la solidaridad, la equidad y la protección de derechos inalienables en los procesos participativos en salud, las motivaciones internas, la cultura y la educación, entre otros aspectos, son también conducentes a la definición de un modelo participativo, condicionado a su vez por un contexto de relaciones asimétricas y un entorno social de características complejas (117). De estos dos flujos surge a modo de vector un valor o grupo de valores dominantes que da encuadre al modelo participativo (118).

Según Arnstein (119), la variable clave es el poder y los niveles de participación reflejan la forma en que se distribuye entre los ciudadanos. Así mismo, pondera el consenso social como un valor superior - en el tope de la escalera está el control ciudadano, una forma de distribución homogénea de los recursos de poder-, pero ve como posibilidades otras modalidades. Por ejemplo, $l a$ estabilidad social como valor ve en la manipulación de la sociedad y en la participación terapéutica una herramienta útil para lograr sus fines, una herramienta de legitimación hegemónica más que una vocación auténtica de brindar un espacio de decisión compartida. El ciudadano visto como cliente, la participación espontánea no institucionalizada, la participación como mecanismo de legitimación, el control ciudadano, y muchas otras modalidades de participación, no hacen más que reflejar un valor o grupo de valores fruto de esas relaciones de poder que dominan el escenario donde los individuos participan (120-124).

Los valores hegemónicos no solamente determinan el modelo participativo, también brindan el esquema maestro sobre el cual se recuestan las decisiones en salud, pudiendo llegar a ser, en sistemas integrados y estructurados, marcos normativos que alcanzan a todo el territorio $(125,126)$. En los sistemas de salud fragmentados, por el contrario, la heterogeneidad de valores y principios impide esta integración, y si bien pueden existir niveles altos de participación, se coexiste en una sensación de conflicto e inseguridad latente, producto de la confrontación cotidiana de modelos muy diferentes que conviven en un mismo sistema de salud (127-129).

Tesis 9: los liderazgos sólidos en sistemas de salud participativos fortalecen la integración de los sistemas de salud y así mejoran su gobernanza

Pareciese, según algunas experiencias, que la noción de una fuerte participación social implica una disminución de la gobernanza de los sistemas de salud y a su vez una devaluación de las capacidades gerenciales y posibilidades de liderazgo en los sistemas de salud, debido a una disminución de su integración vertical (105, 130-135).

Los sistemas de salud tienen una naturaleza productiva - producen servicios de salud-, por lo cual no pueden escapar de su esencia como organizaciones, donde la gerencia y el liderazgo son elementos fundamentales para un correcto desempeño, especialmente en un sector donde existe una demanda de cambio constante (136139). Vale preguntarse entonces de qué manera pueden convivir un liderazgo eficaz 
y una participación social que proporcione valor, respetando la premisa de que un liderazgo eficaz es un factor determinante para la integridad (vertical) del sistema (140). La Administración propone soluciones a este dilema, mediante escuelas que acuden a una actitud especulativa del líder ante la posibilidad de abrir canales participativos, como la teoría de la contingencia (141), o con un modelo más asociado a la cultura y a ver las organizaciones como sistemas abiertos donde debe incorporarse al "otro" como un proceso en continua construcción $(142,143)$.

La falta de un liderazgo claro no es solamente un menoscabo a la gobernanza y eficacia del sistema para atender los requerimientos de salud, sino que en algunos casos - fundamentalmente en el doble comando- puede ser el causal de una ruptura interior (en el seno del sistema) o exterior (con la sociedad) (144-146).

Por ello, los liderazgos en salud tienen como sentido fortalecer la gobernanza de los sistemas de salud, abogar por un mejor desempeño del sistema y conducir las acciones a una construcción coherente con las aspiraciones sociales. Su legitimidad debe basarse tanto en sus antecedentes como en sus resultados y métodos (147152). Los procesos participativos y el liderazgo en salud deben ser vistos como dos dispositivos que tienen objetivos comunes (p. ej., integración), pero también objetivos particulares (p. ej., en los procesos participativos, fortalecer ciudadanía, y en el liderazgo fortalecer gobernanza), los cuales podrán ser sinérgicos si existe una retroalimentación positiva entre ambos y superan algunos estigmas sociales, como la desconfianza entre sociedad y líderes sanitarios (153).
Tesis 10: la participación social y la integración como factores de cambio de los sistemas de salud pertenecen esencialmente al campo de la praxis

Diversos autores alertan sobre el error de asignar al campo de la teoría fenómenos que deben ser abordados desde la praxis (154157). Cuando se hace mención a sistemas sociales y su función, como es el caso de los sistemas de salud, es innegable que ello encuentra sentido en la necesidad social que justifica su existencia, y por lo tanto desde su génesis los sistemas de salud están destinados a ser reactivos a una realidad exterior que los convoca para que sea modificada. Por ello, la participación social en salud y la fragmentación, como fenómenos de los sistemas de salud, deben ser analizadas desde el campo de la praxis.

No hablar sobre la práctica de la participación social y la integración en salud equivale a no interesarse por que estas sean efectivas, o bien a perpetuar situaciones de contradicción entre teoría y práctica. Es por ello que la intervención o proyectos de cambio proveerán de los fundamentos que legitimen una posición (158). Solamente el ejercicio de prácticas destinadas a corregir la fragmentación a través de la participación social, o asumiendo a esta como fin, brindará el cuerpo teórico (y por qué no la solidez técnica y desarrollos de tecnologías) que sustentará las iniciativas, ya que un sentido inverso implica una reticencia o demora en recorrer el camino entre lo actual y lo deseable (159-161).

Esta afirmación lleva a un terreno donde el conocimiento debería ser generado por el análisis de situaciones concretas, en tanto que la comparación entre diferentes situaciones conduce a la selección de conclusiones que deben ser contrastadas con experiencias futuras. Esta metodología, ampliamente utilizada en la investigación de sistemas de 
salud (sistemas comparados de salud, decisiones basadas en evidencias) sería la clave para generar conocimiento desde el ejercicio de la práctica, un hecho positivo si reconocemos las numerosas experiencias que existen en la actualidad y en las cuales podemos inspirar$\operatorname{nos}(162-164)$.

Tesis 11: la fragmentación y la participación social son por su naturaleza complejas y requieren abordajes interdisciplinarios

La noción de causa en salud se fue modificando, con la intención de poder explicar en forma satisfactoria las complejas relaciones que se presentan en el proceso salud-enfermedad. De ello surgió la necesidad de volver a tener una mirada integradora que se opusiese a las clásicas miradas positivistas que fragmentaron en múltiples partes el conocimiento, las prácticas y las tecnologías; a partir de ahí, se contempla a la salud como un proceso que se desarrolla en múltiples niveles y es fruto a su vez de un recorrido histórico-social que reconoce el papel del medio ambiente (en su sentido más amplio) como determinante del continuo salud-enfermedad $(165,166)$.

La participación social en salud y la fragmentación son emergentes u observables de estos sistemas, por naturaleza complejos — sistemas relacionados con interacciones sociales, o que tienen como objetivos productos socialmente sentidos, y cuya definición evoluciona en el tiempo: salud, educación, cultura, etc. - , por lo cual se necesitan nuevos marcos de abordaje, ya que los actuales parecen en cierta forma haber agotado su capacidad de generar conocimiento (167-169). En ese ámbito, la teoría de los sistemas complejos brinda sólidos argumentos para el uso de las investigaciones interdisciplinarias en nuestro ámbito; sostiene que los abordajes con metodologías interdisciplinarias permitirán dar a luz conocimiento concreto sobre problemas que suceden en sistemas complejos $(170,171)$. En la práctica, abordajes desde diferentes disciplinas de un mismo problema y una posterior integración de conocimientos serán la base por la cual podremos comprender y abordar el observable participación social y fragmentación en salud.

Tesis 12: la participación social en sistemas de salud fragmentados debe ser analizada históricamente, ya que la estructura de ambos se define en la dinámica de su pasado

El carácter polisémico de los términos, la complejidad de su marco de estudio, su juicio valorativo a través del tiempo y lo complejo que resulta poder establecer la finalidad de los procesos participativos en salud y su enfoque integrador, hacen que el análisis histórico sea necesario para poder instituir sentido a su referencia. Una tendencia frecuente en el campo de la salud pública es brindarle un carácter fundacional a todo nuevo desarrollo teórico, con una saturación ulterior del campo de conocimiento con nuevos conceptos (salud para todos, promoción de la salud y hospitales de autogestión son representaciones de discursos que dominaban una época) (172-177). No es el problema de que existan discursos que dominen la escena de un tiempo, el problema es que estos tengan el monopolio de la representación de un momento (179). Esta visión desconoce lo previo y a veces provoca un clima de inestabilidad permanente, lo que en cierta forma atenta contra instituciones sólidamente constituidas, necesarias para brindar las respuestas a problemas socialmente sentidos (179). Por ello, los análisis históricos sociales se vuelven poderosas herramientas que nos brindan autoconocimiento y que, de acompañarse de reflexión crítica, permitirán una mayor capacidad de intervenir en la realidad y de plantear posibles escenarios futuros, así como instituir mayor sentido a las organizaciones y sus acciones. 
La participación social en salud y la fragmentación en salud no deberían ser abordadas únicamente como nuevas tendencias en salud pública, sino como procesos históricamente constituidos, cuyos fundamentos se movilizan en una línea de tiempo, y por lo tanto es ahí donde deben buscarse los sentidos que las definen. El análisis histórico no tiene únicamente un carácter descriptivo, sino que permite identificar momentos cruciales, momentos donde debe o debería originarse cambios, y por otro lado, sería un seguro contra la repetición de errores sistemáticos, una característica de aquellos que no aprendieron de experiencias previas (180-182).

\section{Reflexiones finales}

La participación social en salud y la fragmentación en los sistemas de salud son fenómenos ampliamente estudiados y la bibliografía sigue acrecentándose. Constituyen un campo que continúa generando interés, principalmente entre aquellos preocupados por el análisis de sistemas de salud y su desempeño. Como consecuencia lógica de ese amplio abordaje, podemos objetivar diversos modos de relacionar ambos fenómenos y cómo a través de esa relación compleja se afectan los sistemas de salud, positiva o negativamente. La revisión bibliográfica realizada y el análisis cualitativo del contenido permitieron identificar líneas argumentales concretas (las doce tesis) que contienen buena parte de lo escrito en la actualidad sobre el tema, y esto otorga más especificidad al estudio de la participación social en sistemas de salud fragmentados. Que una relación compleja pueda ser definida en sus múltiples dimensiones, con líneas argumentativas razonablemente sólidas, es un objetivo en sí mismo y propicia que la metodología pueda ser utilizada de manera similar para otras relaciones de características similares (por ejemplo, la participación en salud de personas en situación de vulnerabilidad, o la continuidad de servicios en sistemas de salud fragmentados).

Como debilidad, podemos mencionar que quizás un trabajo interdisciplinario con los mismos objetivos de estudio permitiría conclusiones más sólidas o una verificación de las tesis propuestas desde un lugar de mayor legitimidad que el desarrollado por un único autor. Por ello, este trabajo no se propone ser de referencia en el conocimiento sobre el tema, sino más bien un iniciador de conocimientos más integrados, lo cual es en sí la esencia más profunda que llevó a realizarlo y pone de manifiesto la necesidad de debates con marcos de conocimiento más integrales. También es necesario mayor cantidad de estudios de carácter empírico, ya que mucha de la bibliografía consultada sobre el tema es de carácter especulativo o sesgada sobre preconceptos no siempre comprobados, lo cual muchas veces invalida o disminuye la validez de las conclusiones que se generan en estos estudios.

Por último, es necesario en esta época donde lo complejo emerge con mayor frecuencia, poder volver a la esencia del abordaje de los sistemas de salud. Quizás la mayor fortaleza de este trabajo sea volver a poner en discusión que los sistemas de salud son parte de un sistema social y que su existencia se justifica en responder a las necesidades sociales en salud, por lo cual cualquier análisis que se realice debería tener como propósito un elemento en común: el aporte que realiza para mejorar la salud de los miembros de una sociedad. En este caso, brindando líneas argumentativas que sean herramientas para un abordaje más efectivo de la realidad; una especie de hoja de ruta para aquellos gestores que estén firmemente decididos a implementar procesos participativos y sean conscientes de las dificultades que surgen en los sistemas de salud fragmentados para mejorar la salud de los miembros de una sociedad. 


\section{Referencias bibliográficas}

1. Murray CJ, Frenk J. Un marco para evaluar el desempeño de los sistemas de salud. Bulletin of the World Health Organization. 2000; 78 (6): 717-31.

2. Gonzalo G. Los sistemas locales de salud: una transición a la respuesta social organizada. Salud Pública de México. 1991; 33 (6): 617-22.

3. Tobar F. Historia de la demanda en salud [Internet]; 2010 [acceso: julio del 2013]. Disponible en: http:// www.federicotobar.com.ar/eco_10.htm.

4. Vega-Franco L. Ideas, creencias y percepciones acerca de la salud. Reseña histórica. Salud Pública de México. 2002; 44 (2): 258-65.

5. Frenk J. La respuesta social organizada: los sistemas de salud. En: Frenk J. La salud de la población, hacia una nueva salud pública. México, D.F.: Fondo de Cultura Económica; 1994.

6. Organización Mundial de la Salud (oms). Informe sobre la salud en el mundo 2008: La Atención primaria más necesaria que nunca. Ginebra: oms; 2008.

7. Tobar F, Olaviaga S, Solano R. Complejidad y fragmentación: las mayores enfermedades del sistema sanitario argentino. Documento de Políticas Públicas/Análisis N ${ }^{\circ} 108$. Buenos Aires: CIPPEC [Internet]; 2012. Disponible en: http://www.fmed.uba.ar/depto/ saludpublica/108\%20DPP\%20Salud,\%20Complejidad $\% 20 \mathrm{y} \% 20$ fragmentacion, $\% 20$ Tobar, $\% 20$ Olaviaga \%20y\%20Solano, \%202012[1].pdf.

8. Barrillas E. La fragmentación de los sistemas nacionales de salud. Rev Panam Salud Pública. 1997; 1(3): 246-9.

9. Kruse J. Fragmentation in us Medical Education, Research and Practice. Fam Med. 2013; 45 (1): 54-7

10. Stange K. The problem of fragmentation and the need for integrative solutions. Ann Fam Med. 2009; 7 (2): 100-3.

11. McInyire D, Garshong B, Mtei G, Meheus F, et al. Beyond fragmentation and towards universal coverage: insights from Ghana, South Africa and the United Republic of Tanzania. Bulletin of the World Health Organization. 2008; 86 (11): 871-6.

12. Organización Panamericana de la Salud (ops). Redes Integradas de Servicios de Salud. Conceptos, opciones de política y hoja de ruta para su implementación en las Américas. Washington, DC: OPS, Serie: La renovación de la Atención Primaria en Las Américas; 2010.

13. Organización Panamericana de la Salud (OPS). Redes Integradas de Servicios de Salud. Conceptos, opciones de política y hoja de ruta para su implementación en las Américas. Washington, DC: OPS, Serie: La renovación de la Atención Primaria en Las Américas N4; 2008.

14. Elhauge, E. The Fragmentation of U.S. Health Care. Causes and Solution. Londres: Oxford University Press; 2010.

15. Zunzunegui Pastor MV, Lázaro y de Mercado P. Integración y fronteras entre la atención sanitaria y social. Informe Sespas 2008. Gac Sanit 2008; 22 (Supl. 1): 156-62.
16. Gerves J. La gestión de casos y enfermedades y la mejora de la coordinación de la atención sanitaria en España. Informe Sespas 2008. Gac Sanit 2008; 22 (Supl. 1): 163-8.

17. Misseri L. Microutopismo y fragmentación social: Nozick, Iraburu y Kumar. En-claves del pensamiento [Internet]. 2011; 5 (10): 75-88.

18. Paganini JM, Prascilio H. Los sistemas locales de salud: un camino hacia la equidad. La Plata, Argentina: Publicación InUs, Facultad de Ciencias Médicas-unlP; 2000.

19. Bronfman M, Gleizer M. Participación comunitaria: ¿Necesidad, excusa o estrategia? O de qué hablamos cuando hablamos de participación comunitaria. Cad Saúde Públ. 1994; 10 (1): 111-22.

20. Candal LM. La bioética como campo de la participación colectiva, pública e informada. Una propuesta de salud pública. Revista de la Asociación Médica Argentina. 2009; 122 (2): 23-9.

21. Restrepo Vélez O, Vega R. Participación social y comunitaria en Atención Primaria-APs. Rev Gerenc Polit Salud. 2009; 8 (16): 153-64.

22. Barten F. Comentario sobre investigación en sistemas de salud, Atención Primaria de la Salud y participación para la transformación social. Saude en Debate. 2012; 36 (94): 348-51.

23. Ferrer H, Borroto Zaldívar T, Sánchez M, Álvarez Vázquez J. La participación de la comunidad en salud. Rev Cubana Med Gen Integr [Internet]. 2001 [acceso: 10 de junio de 2014]; 17 (3). Disponible en: http://scielo.sld.cu/scielo.php?pid=S086421252001000300011\&script $=$ sci_arttext.

24. Organización Mundial de la Salud (oms). Declaración de Alma Ata. Conferencia Internacional Sobre Atención Primaria de la Salud Alma-Ata. Ginebra: oms; 1978.

25. Organización Panamericana de la Salud (ops). Fundamentos de la renovación conceptual. En: La salud pública de las Américas, nuevos conceptos, análisis de desempeño y bases para la acción. Washington, D.C.: ops; 2002. p. 35-48.

26. Organización Mundial de la Salud (oms). Carta de Ottawa. Primera Conferencia Internacional Sobre Promoción de la salud-oms. Ottawa: oms; 1986.

27. Parsons T. El sistema social. Madrid: Alianza; 1951.

28. Cohen D. Tres lecciones sobre la sociedad postindustrial. Buenos Aires: Katz; 2008.

29. Ottone C, Sojo A, coordinadores. Cohesión social, inclusión y sentido de pertenencia en América Latina y el Caribe. Santiago de Chile: Cepal; 2007.

30. Hopenhaym M, Sojo A, compiladores. Sentido de pertenencia en sociedades fragmentadas: América Latina. Buenos Aires: Siglo 21 Editores; 2008.

31. Jiménez-Martin S, Vilaplana Prieto C. La interacción del sistema social y sanitario. Informe Sespas 2012. Gac Sanit. 2012; 26 (Supl.): 124-33.

32. Hersch P. Participación social en salud: espacios y actores determinantes. Salud Pública de Mex. 1992; 34 (6): 678-88.

33. Ugalde A. Las dimensiones ideológicas de la participación comunitaria en salud en Latinoamérica. En: Menéndez E, Spinelli H. Participación social. 
¿Para qué? Buenos Aires: Lugar Editorial; 2006. p. $19-50$.

34. Menéndez E. Las múltiples trayectorias de la participación social. En: Menéndez E, Spinelli H. Participación social. ¿Para qué? Buenos Aires: Lugar Editorial; 2006. p. 51-80.

35. Sanabria, Ramos, G. Participación social y sanitaria, reflexiones. Rev Cubana Salud Pública. 2001; 27 (2): 89-95.

36. Delgado G, Vázquez Navarrete M, Zapata Bermúdez Y, García M. Participación social en salud: conceptos de usuarios, líderes comunitarios, gestores y formuladores de políticas en Colombia: Una mirada cualitativa. Rev Esp Salud Pública 2005; 79 (6): 697-707.

37. Armitage G, Suther E, Oelke N, Adair C. Health System Integration: state of evidence. Int $\mathrm{J}$ of Integr Care [Internet]. 2009 [acceso: $1^{\circ}$ de julio del año 2014]; 9: 1-11. Disponible en: http://www. ncbi.nlm.nih.gov/pmc/articles/PMC2707589/pdf/ ijic2009-200982.pdf.

38. Salgado-de Snijder VN, Díaz-Pérez MJ, GonzálezVázquez T. Modelo de integración de recursos para la atención de la salud mental en la población rural de México. Salud Pública Mex. 2003; 45: 19-26.

39. Corrales-Nevado D, Alonso-Babarro A, Rodríguez-Lozano MA. Continuidad de cuidados, innovación y redefinición de papeles profesionales en la atención a pacientes crónicos y terminales. Informe Sespas 2012. Gac Sanit. 2012; 26 (Supl.): 63-6.

40. Vázquez ML, Vargas I, Nuño R, Toro N. Organizaciones sanitarias integradas y otros ejemplos de colaboración entre proveedores. Informe Sespas 2012. Gac Sanit. 2012; 26 (Supl.): 94-101.

41. Beland F, Hollande M. Integrated models of care delivery for the frail elderly: international perspectives. Gac Sanit. 2011; 25 (Supl.): 138-46.

42. Suter E, Oelke N, Adair C, Wadell C, et al. Health System Integration: Definition, Process, Impact: A Research Synteshis. Calgary: Alberta Health Service [Internet]; 2007 [acceso: 15 de abril del 2014]. Disponible en: http://www.albertahealthservices.ca/researchers/if-res-hswru-hs-integrationreport-2007.pdf.

43. Valentijn P, Sanneke S, Opheij W, Bruijnzeels M. Understanding Integrated Care: a comprehensive conceptual framework based on integrative function of primary care. Int $\mathrm{J}$ of Integr Care [Internet]. 2013 [acceso: 10 de abril del 2014]; 13: 1-12. Disponible en: https://www.ijic.org/index.php/ ijic/article/view/886/1979.

44. Ledesma Castelltort A. ¿Autogestión o autonomía de gestión? Informe Sespas 2012. Gac Sanit. 2012; 26 (Supl.); 57-62.

45. Labrador J. El mercado fragmenta la salud en EEUU, ¿pero acaso el Estado la integra en Europa? La Biblioteca Cochrane Plus [Internet]. 2013. Disponible en: http://www.bibliotecacochrane.com/BCPGetDocument.asp?SessionID = 8385807\&DocumentID=GCS37-23.

46. Lavandez F, Schwab N, Straatman H. Redes públicas descentralizadas y comunitarias de salud en Bolivia. Rev Panam Salud Pública. 2001; 9 (3): 182-9.
47. Delgado Gallego M, Vásquez-Navarrete L. Barreras y oportunidades para la participación social en Salud en Colombia: percepciones de los actores principales. Rev Salud Pública. 2006; 8 (2): 128-40.

48. Argyris C. Conocimiento para la acción. Una guía para superar los obstáculos del cambio en la organización. Buenos Aires: Granica; 2009.

49. Senge P, Robert C, Ross R, Smith B, Kleiner A. La Quinta Disciplina en la práctica. Buenos Aires: Granica; 2010.

50. Pérez Lueño A. Ciudadanía y definiciones. Alicante: Espagrafic; 1989.

51. Ely Yamin A, Gloppen S. La lucha por los derechos a la salud. ¿Puede ser la justicia una herramienta para el cambio? Buenos Aires: Siglo XXI Editores; 2013.

52. Ronconi L. Derecho a la salud: un modelo para la determinación de contenidos mínimos y periféricos. Salud Colectiva. 2012; 8 (2): 131-49.

53. Kliksberg B. Seis tesis no convencionales sobre participación. Revista de Estudios Sociales [Internet]. 1999 [acceso: 7 de abril de 2014]; 4: 1-19. Disponible en: http://www.redalyc.org/articulo. oa? id $=81511266010$.

54. Programa de las Naciones Unidas para el Desarrollo (PNUD). El sistema de salud argentino y su trayectoria a largo plazo: logros alcanzados y desafíos futuros. Buenos Aires: PNUD; 2011.

55. Parisi E. Participación política, manifestaciones culturales y mecanismos de resistencia. Psicología Política. 2012; 12 (23): 41-8.

56. Sánchez Gómez OA. Los nuevos movimientos sociales. En: Sánchez Gómez OA. Nuevas formas de resistencia civil de lo público a lo privado, movilizaciones de la ruta pacifica 1996-2003 [Internet]. Bogotá: Buenas Prácticas para Superar el Conflicto-Pnud; 2006: p. 39-62. Disponible en: http://www.saliendodelcallejon.pnud.org.co/ Los_Nuevos_Movimientos_Sociales.pdf.

57. Henao Escovar J, Eugenia Pinilla V. Jóvenes y ciudadanía en Colombia: entre la politización social y la participación institucional. Rev Latinoam Cienc Soc Niñez Juv. 2009; 7 (2): 1405-37.

58. Celedón C, Noé M. Reforma del sector salud y participación social. Rev Panam Salud Pública. 2000; 8 (1/2): 99-104.

59. Uriarte E. Diálogo social, teoría y práctica. Boletín Técnico Interamericano de Formación Profesional. Diálogo Social en Uruguay. 2007; 157: 11-26.

60. Ruiz Azarolla A, Perestero Pérez L. Participación ciudadana en salud: formación y toma de decisiones compartidas. Informe Sespas 2012. Gac Sanit. 2012; 26 (Supl.): 158-61.

61. Briceño León R. El contexto político de la participación comunitaria en América Latina. Cad Saude Pública. 1998; 14 (Supl. 2): 141-7.

62. Adolfo Gómez C. Influencia de los grupos de interés y asociación en las reformas y sistemas de salud. Revist Gerenc Politic de Salud, 2005; 9: 62-80.

63. Uribe Gómez M. La dinámica de los actores en la implementación de la reforma del Sistema de Salud en Colombia: exclusiones y resistencia (1995-2003). Revist Gerenc Politic de Salud. 2009; 8 (17): 44-68. 
64. Vargas Forero R. Hacia una teoría del capital social. Revista de Economía Institucional. 2002; 4 (6): 71-108.

65. Ramírez Plascencia J. Tres visiones sobre el capital social: Bourdieu, Coleman y Putnam. Republicana: Política y Sociedad. 2005; 4 (4): 21-36.

66. Ríos Cazarez A, Ríos Figueroa J. Capital social y democracia: una revisión crítica de Putnam. Política y Gobierno. 1999; 6 (2): 513-28.

67. Durston J. Qué es el capital social comunitario [Internet]. Santiago de Chile: Naciones Unidas-Cepal Serie Políticas Sociales $N^{\circ} 38 ; 2000$. Disponible en: http://www.eclac.org/publicaciones/xml/5/4885/ lcl1400.pdf.

68. Putnam R. Social capital, measurements and consequences [Internet]. Washington D.C.: OCDE, 2000. Disponible en: https://www1.oecd.org/edu/ innovation-education/1825848.pdf.

69. Atria R, Salies M, compiladores. Capital social y reducción de la pobreza en América Latina: en busca de un nuevo paradigma. Santiago de Chile: Cepal, Naciones Unidas, Universidad del Estado de Michigan; 2003.

70. García-Londoño J. Alcances y limitaciones teóricas y prácticas del "capital social en salud". El caso del sistema de salud de Medellín. Revist Gerenc Politic de Salud. 2013; 12 (24): 184-208.

71. Etkin J. Capital social y valores en la organización sustentable. El deber ser, poder hacer y la voluntad creativa. Buenos Aires: Granica; 2007.

72. Veenstra G. Social Capital, SES, and Health: an individual level analysis. Social Science and Medicine. 2000; 50 (5): 619-29.

73. Macinko J, Starfield B. The Utility of Social Capital in Research on Health Determinants. The Mildbank Quarterly. 2001; 79 (3): 387-427.

74. Fijiwara T, Kawachi I. Social Capital and Health. A study of adult twins in U.S. Am J Prev Med. 2008; 36 (2); 139-144.

75. Sapag JC, Kawachi I. Capital social y promoción de la salud en América Latina. Revista Saude Publica. 2007; 41 (1): 139-49.

76. Kripper CE, Sapag JC. Capital social y salud en América Latina y el Caribe: una revisión sistemática. Rev Panam Salud Pública. 2009; 25 (2): 162-70.

77. Belmartino S. Modelo médico hegemónico (ponencia). Ponencia Jornadas de A.P.S.-CONAMERA.R.H.N.R.G [Internet]. 1988; 198-211. Disponible en: http://www.unla.edu.ar/documentos/institutos/ isco/cedops/libro1a13.pdf.

78. Menéndez EL. Las crisis del modelo médico hegemónico y las alternativas auto gestionadas en Salud. Cuadernos Médicos Sociales [Internet]. 1982 [acceso: 10 abril del 2014]; 21: 1-13. Disponible en: http:// www.ilazarte.com.ar/cuadernos/pdf/n21a071.pdf.

79. Gracia D. Ética profesional y ética institucional: ¿Convergencia o conflicto? Rev Esp Salud Pública. 2006; 80 (5): 457-67.

80. Ortiz Pommier A. Gestión clínica y conflicto de intereses. Acta Bioethica. 2009; 15 (2): 157-64.

81. Vargas Hernández J. Análisis de los fundamentos de la teoría institucional. Revista Digital Universitaria. 2005 [acceso: 30 de junio del 2014); 6 (8):
1-21. Disponible en: http://www.revista.unam.mx/ vol.6/num8/art84/ago_art84.pdf.

82. Molteni G. Principales aportes de la Nueva Economía Institucional y sus críticos. Revista Valores de la Sociedad Industrial. 2006; 24 (67): 8-17.

83. Martín J, López del Amo GM. Incentivos e instituciones sanitarias públicas. Granada: Escuela Andaluza de Salud Pública; 1994.

84. Parada J. Economía Institucional original y Nueva Economía Institucional: semejanzas y diferencias. Revista de Economía Institucional. 2003; 5 (8): 92-116.

85. Rodríguez M. El poder de voto en el Consejo Nacional de Seguridad Social en Salud. Revista de Economía Institucional. 2007; 9 (16): 223-53.

86. Echeverry-López ME. Las organizaciones de usuarios, incidiendo en el derecho a la salud. Rev Gerenc Politic Salud. 2009; 8 (17): 80-105.

87. Haro-Honrubia A. Antropología del conflicto, reflexiones sobre el mundo global. Convergencia, Revista de Ciencias Sociales. 2012; 60: 177-204.

88. Sen A. La violencia de la ilusión. En: Sen A. Identidad y violencia, la ilusión del destino. Buenos Aires: Katz Editores; 2007.

89. Torrijos Rivera V. Pérez Carbajal A. De los conflictos complejos: naturaleza, estructura y morfología de los conflictos intratables e imperecederos. Rev Relac Inst Estrat Segur. 2013; 8 (2): 119-42.

90. Aristóteles. La Política. Buenos Aires: La Editorial Virtual; 2007.

91. Wendhausen AL. Relaciones de poder y democracia en los consejos de salud de Brasil: estudio de caso. Rev Esp Salud Pública. 2006; 80 (6): 697-704.

92. Millán Valenzuela H. La democracia secuestrada. Actores sociales y representatividad en México. Perfiles Latinoamericanos. 2012; 40: 165-204.

93. Etvitty J, Leyva R. Los actores políticos y sociales en la formulación de la reforma sanitaria en España. Cad Saude Pública. 1995; 11 (1): 34-44.

94. Vidella G. Democracia: ¿razones o pasiones? Tópicos. 2013; 25: 63-80.

95. Galafassi G. Para una relectura de los procesos de conflicto y movilización social en la Argentina de inicios de milenio (2001-2003). Revista Mexicana de Sociología. 2012; 74 (1): 69-98.

96. Leiras M, Cruzalegui I. Argentina: Problemas macroeconómicos, conflicto social y debilitamiento de la coalición de gobierno. Revista de Ciencia Política. 2009; 29 (2): 223-46.

97. Pereira-Menaut AC. Doce tesis sobre la política. México: Universidad Nacional Autónoma de México; 2008.

98. Espinoza M. La participación social como una relación socio-estatal acotada por la concepción de democracia y ciudadanía. Andamios. 2009; 5 (10); 71-109.

99. Carrillo AM. Repensando la relación entre democracia y conflicto: algunas propuestas para ampliar el consenso democrático. Perfiles Latinoamericanos. 2013; 41: 45-69.

100. García Leal L. Diversificación de las formas de resolución de conflictos como política pública. Fronesis, 2008; 15 (1): 96-115. 
101. López de Lizaga L. Diálogo y conflicto: la crítica de Carl Schmidt al liberalismo. Dianoia. 2012; 77 (68): 113-40.

102. Berardo R, Mazzalay V. Confianza, influencia política e intercambio de recursos en arenas decisorias regionales. Revista de Ciencia Política. 2012; 32 (2): 479-500.

103. Vásquez ML, Siqueira E, Kruze I, Da Silva A, Leite IC. Los procesos de reforma y la participación social en salud en América Latina. Gac Sanit. 2002; 16 (1).

104. Nigenda López G, Juárez Ramírez C, Ruiz Larios J, Herrera C. Participación social y calidad de los servicios de salud: experiencia del aval ciudadano en México. Rev Saude Publica 2013; 47 (1): 44-51.

105. Arredondo-López A, Orozco Núñez E, Castañeda X. Gobernanza en sistemas de salud y participación social en México a partir de la reforma de salud. Hitos de Ciencias Económicas Administrativas. 2005; 11 (29): 15-22.

106. Echeverry López ME. Balance de los procesos de descentralización y participación social en salud en Colombia en la década de los 90. Revista Facultad Nacional de Salud Pública. 2000; 18 (2): 19-40.

107. Abrantes Pego R. Participación social en salud: un estudio de caso en Brasil. Salud Pública Mex. 1999; 41 (6): 466-74.

108. Mercer H, Ruiz VA. Participación de organizaciones comunitarias en la gestión de salud: una evaluación de la experiencia del Programa UNI. Interface-Comunic, Saude, Educ. 2004; 8 (15): 289-302.

109. Ávila Urdaneta M. La salud en el paradigma de la descentralización en América Latina. Revista de Ciencias Sociales, 2010; 16 (3): 526-43.

110. Orozco Núñez E, González Block MA, Kageyama-Escobar LM, Hernández Prado B. Participación social en salud: la experiencia del programa de salud maternal. Arranque parejo en la vida. Salud Pública Mex. 2009; 51 (2): 104-13.

111. Bossert TJ, et al. La descentralización de los servicios de salud en Bolivia. LACHSR initiative Health Product Inventory. 2000. № 35 [Internet]. Disponible en: http://www2.paho.org/hq/ dmdocuments/2010/35-Descentralizacion_Servicios_Salud-Bolivia.pdf.

112. Méndez CA, Vanegas López JJ. La participación social en salud: el desafío en Chile. Rev Panam Salud Pública. 2010; 27 (2): 144-8.

113. Montenegro H, et al. Combating Health Care Fragmentation through integrated health service delivery networks in the Americas: lessons learned. Int J of Integr Care. 2011; 19 (5).

114. Ferrada O, Méndez C. Implementación de las redes asistenciales de salud en Chile: percepciones de los profesionales de la salud. Rev. Gerenc. Polit. Salud. 2013; 12 (24): 100-13.

115. Organización Mundial de la Salud. Integrated Health Services - What and Why [Informe]. - Ginebra: OMs; [Internet] 2008. Disponible en: http:// www.who.int/healthsystems/technical_brief_final. pdf.
116. Sollazo A, Berteretche R. El sistema nacional integrado de Salud en Uruguay y los desafíos para la Atención Primaria. Ciencia \& Saude Coletiva. 2011; 16 (6): 2829-40.

117. Cárdenas Gracia J. El modelo deliberativo participativo. Cuestiones Constitucionales. 2004; 11: 55-89.

118. Guillen A, Sáenz K, Badii MH, Castillo J. Origen, espacio y niveles de participación ciudadana. Daena: International Journal of Good Conscience; 2009: 179-93.

119. Arnstein S. A ladder of citizen participation. JAIP. 1969; 35 (4): 216-22. Disponible en: https:// www.planning.org/pas/memo/2007/mar/pdf/ JAPA35No4.pdf.

120. Kliksberg B. Estrategias y metodologías para promover la participación social [ponencia]. Conferencia Mundial Sobre Determinantes Sociales de la Salud. Brasil [Internet]; 2011. Disponible en: http://redgobiernoabierto.org/wpcontent/uploads/2012/12/Kliksberg-Estrategias-yMetodologías.pdf.

121. Menéndez E. Participación social en salud como realidad técnica y como imaginario social privado. Cuadernos Médicos Sociales. 1998; 73: 5-22.

122. Rentería Rodríguez M. Tipos y modos de la participación ciudadana en los procesos de ordenamiento del territorio: dos casos para su análisis. En: Aguilar Robledo M, et al. Ordenamiento territorial y participación social: problemas y desafíos. México D. F.: Semarnat; 2012.

123. Martín-García M, Pontte-Mittelbrum C, Sánchez Bayle M. Participación social y orientación comunitaria de los servicios de salud. Gac Sanit. 2006; 20 (Supl. 1): 192-202.

124. ISalud. Investigación participación en salud Mercosur (2006-2008). Psicología y Psicopedagogía. Publicación Virtual de la Facultad Virtual de Psicopedagogía de la Universidad del Salvador. 2009; 20. Disponible en: http://www.usal.edu. ar/archivos/psico/otros/participacion_ciudadana_en_salud_canay_y_otros.pdf.

125. Sánchéz Martínez F, Abellan Perpiñán J, Martínez Pérez J. ¿Cómo se deben establecer y evaluar las prioridades en salud y servicios de salud? Métodos de priorización y disparidades regionales. Informe Sespas 2008. Gac. Sanit. 2008; 22 (Supl.): 126-36.

126. Harper S, King NB, Meersman SC, Reichman ME, Breen N, Linch J. Juicios de valor implícitos en la medición de las desigualdades de salud (traducción oficial del original). Rev Panam Salud Pública. 2014; 35 (4): 293-304.

127. Godwin N. Taking integrated care forward: the need for shared value. Int J Integr Care. 2013; 13: 1-2.

128. Yavich N, Bascolo EP, Haggerty J. Construyendo un marco de evaluación de la Atención Primaria de la Salud. Salud Pública Mex. 2010; 52 (1): 39-45.

129. Cetrangolo, O. Devoto F. Organización de la salud en Argentina y equidad, una reflexión sobre las reformas de los años noventa e impacto de 
la crisis actual [Internet]; 2002. Disponible en: http://www.eclac.cl/publicaciones/xml/1/13041/ SALUDEQYREF.pdf.

130. Artaza-Barrios O, Toro-Devia O, FuentesGarcía A, Alarcón Hein A, Arteaga-Herrera O. Gobiernos de redes asistenciales: evaluación de los Consejos Integradores de la Red Asistencial (CIRA) en el contexto de la reforma de salud en Chile. Salud Pública Mex. 2013; 55 (6): 650-8.

131. Cunill Grau N, Fernández MM, Vergara M. Gobernanza sistémica para un enfoque de derechos de salud. Un análisis a partir del caso chileno. Salud Colectiva. 2007; 7 (1): 21-33.

132. Delgado-Gallego M, Vázquez-Navarrete ML. Conocimientos, opiniones, y experiencias en la aplicación de las políticas de participación en Salud en Colombia. Rev. Salud Pública. 2006; 8 (3): 150-67.

133. Stolkiner A, Comez Y, Garbus P. Alcances y potencialidades de la Atención Primaria de la Salud en Argentina. Ciencia \& Saude Colectiva. 2011; 16 (6): 2807-16.

134. Bascolo E, Yavich N. Gobernanza del desarrollo de la APs en Rosario, Argentina. Rev Salud Pública. 2010; 12 (1): 89-104.

135. Roth Deubel A, Molina Martin G. Rectoría y gobernanza en salud pública en el contexto del sistema de salud colombiano, 2012-2013. Rev Salud Pública. 2013; 15 (1): 44-55.

136. Bursztyn I, Kushnir R, Giovanella L, Stolkiner A, et al. Notas para el estudio de la Atención Primaria en contextos de sistemas de salud segmentados. Rev Salud Pública. 2010; 12 (1): 77-88.

137. Milton T. Epidemiology and leadership in Public Health. Epidemiol Bull. 1989; 10 (1): 9-13.

138. Neri A. Liderazgo en los servicios de salud. Educ Med Salud. 1986; 20 (4): 442-7.

139. Ferreira P. El liderazgo en salud pública en Latinoamérica. Educ Med Salud. 1989; 23 (1): 5-13.

140. Denis J. Lamothe L, Langley L, Guerad S. Reforma y gobernanza en salud: el atractivo del gerenciamiento en la acción pública. Rev Salud Pública. 2010; 12 (1): 105-22.

141. Robbins H, Finley M. Visión defectuosa. Si no sabes adónde vas... es probable que llegues ahí. En: Robbins H, Finley M. Por qué fallan los equipos. Los problemas y como corregirlos. Buenos Aires: Granica; 2008. p. 139-42.

142. Robbins S, Coulter M. Liderazgo. En: Robbins S, Coulter M. Administración, octava edición. México: Pearson Education; 2005.

143. Mintzberg H. Safari a la estrategia: una visita guiada por la jungla del management estratégico. Primera edición, tercera reimpresión. Buenos Aires: Granica; 2008.

144. Dogson R, Kelley L, Drager N. Global Health Governance: A conceptual review. Discussion Paper $\mathrm{N}^{\circ} 1$. Ginebra: London School of Hygiene and Tropical Medicine, Centre on Global Change and Health, wHO, Dept. Of Health and Development [Internet]; 2002. Disponible en: http://libdoc.who. int/publications/2002/a85727_eng.pdf.
145. Hoyos Hernández PA, Cardona Ramírez MA, Correa Ramírez D. Humanizar los contextos de salud: una cuestión de liderazgo. Invest Educ Enferm. 2008; 26 (2): 218-25.

146. Spinelli H. El proyecto político y las capacidades de gobierno. Salud Colectiva. 2012; 8 (2): 107-30.

147. Rodríguez C, Lamothe L, Bartten F, Haggerty J. Gobernanza y salud: significado y aplicaciones en América Latina. Rev Salud Pública. 2010; 12 (1): 151-9.

148. Hufty M. Gobernanza en salud pública: hacia un marco analítico. Rev Salud Pública. 2010; 12 (1): 39-61.

149. Hufty M, Bascolo E, Bazzani R. Gobernanza en salud: un aporte conceptual y analítico para la investigación. Cad Saude Pública. 2006; 22 (Supl.): 35-45.

150. Bascolo E. Gobernanza y economía política de la APS en Argentina. Ciencia \& Saude Colectiva. 2011; 16 (6): 2763-72.

151. Bascolo E. Gobernanza de las organizaciones de salud basadas en Atención Primaria de la Salud. Rev Salud Pública. 2010; 12 (1): 8-27.

152. Bazzani R. Gobernanza y salud: aporte para la innovación en sistemas de salud. Rev Salud Pública. 2010; 12 (1): 1-7.

153. Flores W. Los principios éticos y los enfoques asociados a la investigación de la gobernanza en los sistemas de salud: implicancias conceptuales y metodológicas. Rev Salud Pública. 2010; 12 (1): 28-38.

154. Castoriadis C. La institución imaginaria de la sociedad. Buenos Aires: Tusquets; 2007.

155. Foucault M. Un diálogo sobre el poder y otras conversaciones. Buenos Aires: Alianza Editores; 2008.

156. Samaja J. El proceso de investigación y sus dimensiones. En Samaja J. Epistemología y metodología, elementos para una teoría de la investigación científica. Buenos Aires: Eudeba; 1999.

157. López-Moreno A. La teoría de la acción en Weber, Parsons y Habermas: algunas consideraciones críticas. Foro, Nueva Época. 2005; 1: 179-201.

158. Carballeda A. La intervención en lo social: Exclusión e integración en los nuevos escenarios sociales. Buenos Aires: Paidós; 2007.

159. Almeida C. Delimitación del campo de la investigación en sistemas y servicios de salud: desarrollo histórico y tendencias. Texto base para la discusión. En: Almeida C, editor, Stolkiner A, Stermann Heinman L, Bataglia ME, Sánchez D. Investigación en sistemas y servicios de salud. Río de Janeiro: Red de Investigaciones en Sistemas y Servicios de Salud del Cono Sur [Internet]; 2000. p. 11-35. Disponible en: http://www.bireme.br/bvs/ equidad/cuadernos1.pdf.

160. Fee E. Divorce between theory and practice. The system of public health training in the United States. Ciencia \& Saude Coletiva. 2008; 13 (3): 841-52.

161. Onocko Campos R. La praxis, posible hilo de Ariadna en el laberinto tecnológico. En: Onocko Campos R. La planificación en el laberinto: 
un viaje hermenéutico. Buenos Aires: Lugar Editorial; 2007.

162. Frenk J, Gómez-Dantes O. Lecciones globales de la reforma mexicana de salud: empoderamiento a través del uso de evidencia. Rev Peru Med Exp Salud Pública. 2010; 27 (3): 412-18.

163. Abramtes Pego R. Almeida C. Teoría y práctica en las reformas en los sistemas de salud: los casos de Brasil y México. Cad Saude Pública. 2002; 18 (4): 971-89.

164. Pimentel E. El desarrollo sostenible de los sistemas de salud: un paradigma emergente y saludable. Ciencia \& Saude Coletiva. 2003; 8 (1): 243-9.

165. Moiso A. Los determinantes de la salud. En: Barragan HL, director, Moiso A, Mestorino MA, Ojea OA. Fundamentos de salud pública. La Plata, Argentina: Editorial Universidad Nacional de la Plata; 2007; p. 161-190.

166. Ramis Andalia R, Sotolongo Codina PL. Aporte del pensamiento y las ciencias de la complejidad al estudio de los determinantes de la salud. Revista Cubana de Salud Pública. 2009; 35 (4); 65-77.

167. Toro Palacio F, Ochoa Jaramillo F. Salud: un sistema complejo adaptativo. Rev Panam Salud Pública. 2012; 31 (2): 161-5.

168. Hudson C, Vissing Y. Sustainability at the Edge of Chaos: Its Limits and Possibilities in Public Health [Internet]. Biomed Res Int. 2013. Disponible en: http://www.ncbi.nlm.nih.gov/pmc/articles/ PMC3766553/.

169. Ekdahl AW, Hellstrom I, Andersson L, et al. Too complex and time-consuming to fit in. Physicians' experiences of elderly and their participation in medical decision making: a grounded theory [Internet]. BMJ Open. 2012; 2 (3). Disponible en: http://bmjopen.bmj.com/content/2/3/e001063.full.

170. García R. Sistemas complejos: conceptos, método y fundamentación epistemológica de la investigación interdisciplinaria. Barcelona: Gedisa; 2006.

171. Cofiño R, Pasarin MI, Segura A. ¿Cómo abordar la dimensión colectiva de la salud de las perso- nas? Informe Sespas. 2012. Gac Sanit 2012; 26 (Supl.): 88-93.

172. Tobar F. Cambios de paradigmas en salud pública [Internet]. Trabajo presentado en el XII Congreso CLAD, Buenos Aires, 2008. Disponible en: http:// www.fsg.org.ar/maestria_pdfs/3.pdf.

173. Hernández-Girón C, Orozco-Núñez E, Arredondo-López A. Modelos conceptuales y paradigmas en salud pública. Rev Salud Pública. 2012; 14 (2): 315-24.

174. Belmartino S. La atención médica en Argentina. Historia, crisis y nuevo diseño institucional [Internet]. Seminario IV. Ciclo de Seminarios Salud y Política Pública, Cedes, 2005. Disponible en: http://imgbiblio.vaneduc.edu.ar/fulltext/files/ TC063105.pdf.

175. Belmartino S. Contribución al debate metodológico en salud. Salud Colectiva. 2008: 125-31.

176. Belmartino S. Una década de reforma de la atención médica en Argentina. Salud Colectiva. 2005; 1 (2): 155-71.

177. Belmartino S. Los valores vinculados a equidad en la reforma de la atención médica en Argentina. Cad Saúde Pública. 2002; 18 (4): 1067-76.

178. Angenot M. El discurso social, los límites de lo histórico, de lo pensable y lo decible. Buenos Aires: Siglo Veintiuno Editores; 2012.

179. Trouve H, Couturier Y, Etheridge F, Saint-Jean $\mathrm{O}$, et al. The path dependency theory: analytical framework to study institutional integration. Int J of Integr Care. 2010; 10: 1-9.

180. Pierson P, Scokpol T. El institucionalismo histórico en la ciencia política contemporánea. Revista Uruguaya de Ciencias Políticas. 2008; 7-35.

181. De Vos P, Malaise G, De Ceukelaire W, Pérez D, et al. Participación y empoderamiento en la Atención Primaria de la Salud: desde Alma Ata hasta la era de la globalización. Medicina Social. 2009; 4 (2): 127-34.

182. Urbanos R. La salud en todas las políticas. Tiempo de crisis, ¿tiempo de oportunidades? Informe Sespas 2010. Gac Sanit. 2010; 24 (Supl.): 7-11. 